\title{
A commutation strategy for IGBT-based CSI-fed parallel resonant circuit for induction heating application
}

\author{
MOLAY ROY*i] and MAINAK SENGUPTA \\ Department of Electrical Engineering, Indian Institute of Engineering Science and Technology, Shibpur, \\ Howrah 711103, India \\ e-mail: molay.roy@gmail.com; mainak.sengupta@gmail.com; msg@ee.becs.ac.in
}

MS received 29 October 2015; revised 26 March 2016; accepted 20 September 2016

\begin{abstract}
A brief study on a commutation strategy for a current source inverter (CSI)-fed parallel resonant circuit, using switches formed by IGBTs with series diodes, is presented in this paper. The dynamic behaviour of the inverter and different strategies for its reliable operation are discussed here considering parasitic inductance and overlap time between CSI switching devices. The dynamic behaviour of the switches decides the upper frequency limit for the application. IGBTs with the series diodes behave as uni-directional current switches with bi-directional voltage blocking capability. This feature should be taken into account to decide on an appropriate switching strategy for this converter configuration.
\end{abstract}

Keywords. Induction heating; current source inverter (CSI); parallel resonance; IGBT commutation.

\section{Introduction}

In induction heating applications, two inverter topologies might be used: voltage source and current source inverters (CSI). However, due to a number of reasons [1], the CSI configuration lends itself naturally for popular use in induction heating applications. At the output terminals of the CSI a parallel resonant circuit remains connected where the heating coil is in parallel with a compensating capacitor [2-6]. This capacitor effectively supplies the reactive VAr requirement of the coil. The working frequency of the inverter is just above the resonant frequency, which depends on the values of the capacitor and the coil equivalent series $R-L$ parameters. The aim of this paper is to present an experimental study on a commutation strategy for bi-directional voltage blocking with uni-directional currentcarrying capability when used in such a CSI.

Figure 1 shows the CSI configured with IGBTs. The criticalities of the choice of switching frequency and commutation principles have been established later in this paper. A study on the possible switching strategies has been done to prevent overvoltage in the circuit. Finally, a control strategy is proposed and adopted to identify the optimum switching frequency and the overlap time for optimum phase difference (between the inverter terminal current and voltage) at which the switching losses will be minimum. It may also be mentioned here that throughout this text the term switches refers to solid-state switching devices (IGBT), unless otherwise mentioned.

*For correspondence

\section{Operating principles of an IGBT-based CSI}

A parallel circuit fed from an ideal IGBT-based CSI is shown in both figure $1 \mathrm{a}$ and $\mathrm{b}$. Figure $1 \mathrm{a}$ shows the circuit without considering the presence of any parasitic component and figure $1 \mathrm{~b}$ includes that parasitic inductance $\left(L_{p}\right)$ in series with the load-resonant circuit. This inductance arises due to the connections from the inverter terminals to the heating coil. Among all the converter configurations, the $\mathrm{H}$ bridge inverter transfers the maximum active power to the load. Ideally the switches $T_{1}$ and $T_{2}$ and $T_{3}$ and $T_{4}$ commutate alternately with a duty cycle of $50 \%$. The induction coil and workpiece combination is modelled as a constant resistance and inductance in series. An IGBT-based CSI may be used to get the almost unity power factor operation. In case of IGBT-based CSI, series diodes are added to block the reverse voltage across the switch; hence figure 1a and $\mathrm{b}$ shows a diode in series with an IGBT.

The analysis of the commutation process is essential to decide on the details of the switching strategy of the IGBTs for a reliable operation of the CSI. In the present set-up, it has been observed during experiments that the effect of the parasitic inductance associated with the connecting wires (and cables) between the inverter and the resonant circuit is not really negligible. Considering the inductance in the switching model and with an appropriate control strategy, it is possible to keep the current under control during all the switching transitions, thereby avoiding undesirable overvoltage. The optimum switching process can be defined when there is no sudden change in the current flow. This 


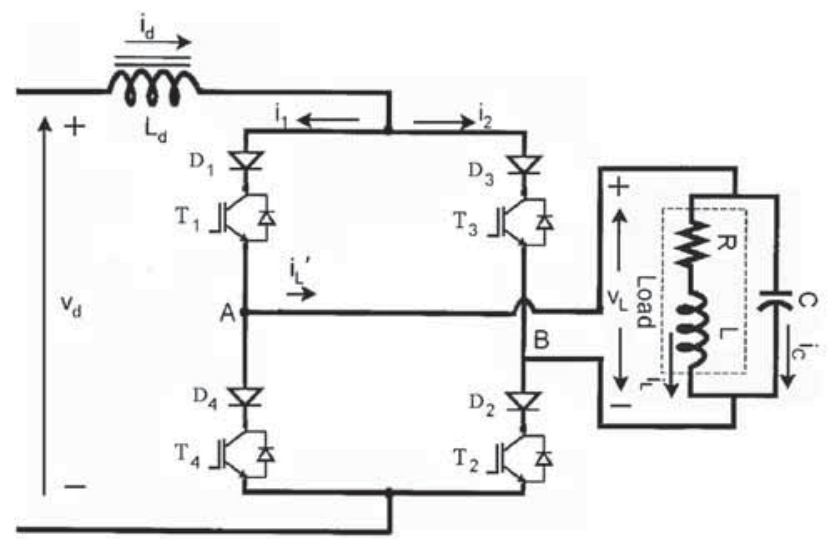

(a)

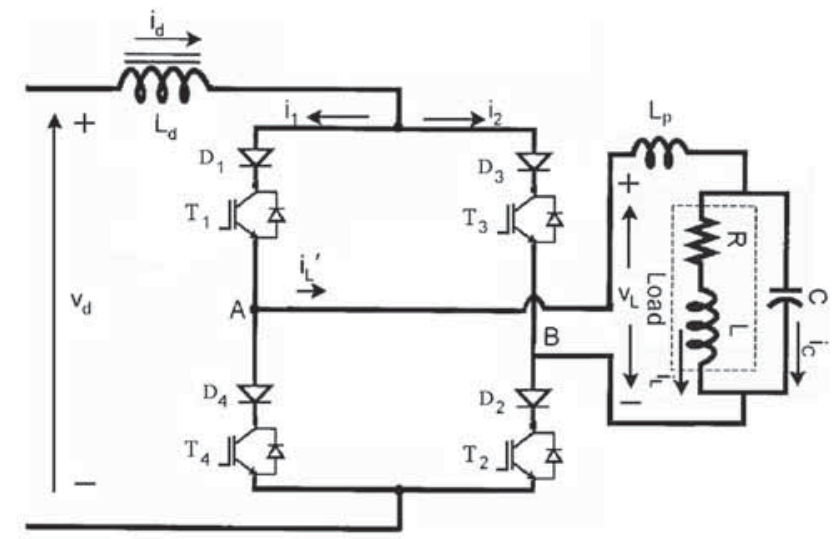

(b)

Figure 1. Circuit diagram of CSI-fed parallel resonant circuit (a) without considering parasitic inductance $\left(L_{p}\right)$ and $(\mathbf{b})$ considering parasitic inductance $\left(L_{p}\right)$.

occurs only when the total overlap time, during which all the switches are on, is co-terminus in time with the current change from $-I_{d}$ to $+I_{d}$, where $I_{d}$ is the magnitude of the idealised level current in the DC link. This duration is the optimum overlap time because it minimises the turn-on switching losses in the IGBTs and no over-voltage is produced. Moreover, if the turn-off matches with the zero crossing of the load voltage $\left(V_{L}\right)$, the turn-off losses are reduced to a minimum. The details are discussed in the following section.

\section{Commutation analysis}

It is known that at resonant frequency the power supplied to the workpiece is maximum. The commutation at resonant frequency is realised with low losses when having high $Q$ (quality factor [7]) values. However, resonant frequency switching is not possible in all control strategies due to the change of parameters (like magnetic permeability, inductance, etc.) during heating. However, the same has been achieved here in an interesting way, which effectively dynamically tracks the changing resonant frequency. This 'dynamic tracking' has been successfully implemented as a part of this experimental work and has been reported elsewhere [8]. In this connection it may be worthwhile to study all the possible operating modes of the converter.

In this section, the modes in which the converter tends to come out of resonance are studied in greater detail. One objective of this study is to find out the sequence of events occurring during the switching transitions and their effects. Finally, the most preferred operating mode for the converter is indicated. The operations are discussed (i) without considering the parasitic components and overlap time of the converter, (ii) without considering the parasitic components, but considering overlap time of the converter and (iii) considering a more realistic situation by including effects of both parasitic inductances and switching overlap time of the converter. The symbols for the different variables used in the explanations below follow those indicated in figure $1 \mathrm{a}$ and $\mathrm{b}$.

It may be noted that, in the case of CSI, two switches of the same inverter leg cannot be opened at the same time (KCL violation). In this case, the overlap time is the interval during which both switches from the same inverter leg are turned on. It may be recalled that [9], only for coils having high $Q$ values $(Q \gg 1)$, resonant frequency is given by

$$
f_{r}=\frac{1}{2 \pi \sqrt{L C}}
$$

in the case of parallel resonance.

\subsection{Resonant switching $\left(f_{s w}=f_{r}\right)$}

Figure 2 shows the appropriate switching sequence considering that the inverter is commutating at zero phase shift between current and voltage, i.e., at resonant frequency (here $Q=14 \gg 1$ ). The commutation in figure $2 \mathrm{a}$, as illustrated by the gate signals, occurs when the voltage is crossing zero. Observing the voltages across $T_{1}$ (and $T_{2}$ ) and $D_{1}$ (and $D_{2}$ ), the voltage (second set of plots from top in figure $2 \mathrm{a}$ ) is blocked by $T_{1}$ (and $T_{2}$ ) for half of the cycle while they are off, whereas the diodes are always forward biased. Under these circumstances, there are two possible states. The first state labelled ' $\mathrm{S}-\mathrm{I}$ ' occurs when $T_{1}$ and $T_{2}$ are conducting and the second ' $\mathrm{S}$-II' when $T_{3}$ and $T_{4}$ are conducting. This case will ensure very low switching losses because the commutation takes place when the current and voltage are both zero.

Next, considering the overlapping of switching signals, figure $2 b$ shows undesirable turn-on and turn-off of the switches (states S-II and S-III), causing high switching 


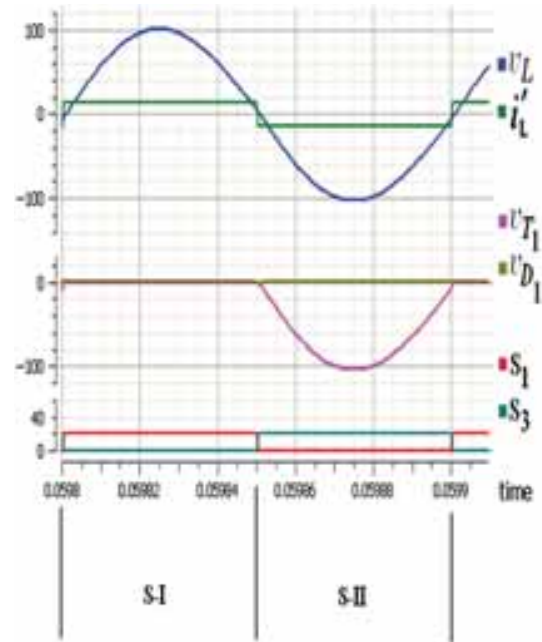

(a)

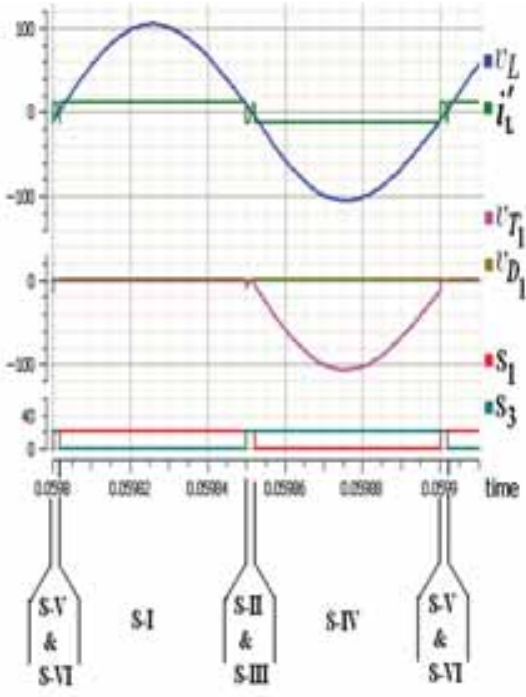

(b)

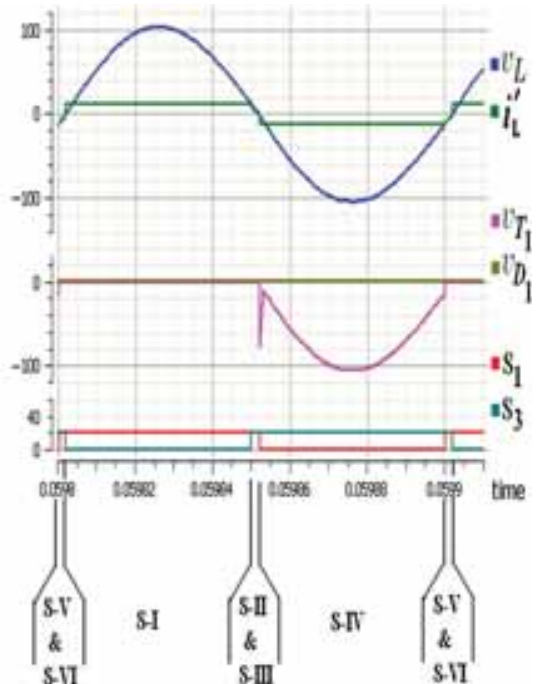

(c)

Figure 2. The waveforms of resonant frequency operation (a) without considering the parasitic components and overlap time, (b) without considering the parasitic components, but considering only overlap time and (c) considering parasitic inductances and overlapping time of the converter.

losses in the converter. Similarly, considering the parasitic inductance $\left(L_{p}\right)$ of the converters, figure $2 \mathrm{c}$ shows that during the transition between S-III and S-IV, the current changes polarity due to the hard turn-off of $T_{1}$ and $T_{2}$, generating a large voltage across the $L_{p}$ due to current variation. This voltage gets added to $v_{L}$ and increases the voltage across switches, which can lead to their destruction.

\subsection{Inductive load switching $\left(f_{s w}<f_{r}\right)$}

Figure 3 shows the waveforms and the corresponding sequence of events for the case of switching below resonant frequency. In this case, this switching is called inductive load switching because the voltage leads the current.

Under these circumstances (second row plots in figure 3), diodes are blocking during a part of the period although, in this case, the blocking occurs during the change of polarity of $v_{L}$ and commutation of the diodes. Observing the sequence of events in figure $3 \mathrm{a}$ and $\mathrm{b}$, the current $i_{L}^{\prime}$ is conducting through $T_{1}$ and $T_{2}$ in state 'S-I'. Then, $v_{L}$ changes polarity, blocking $D_{3}$ and $D_{4}$ with ZCS because the series switches are not conducting (S-II). After this, $T_{3}$ and $T_{4}$ are turned on with ZVS because series diodes $\left(D_{3}\right.$ and $D_{4}$ ) are blocking the load voltage (S-III). Finally, $T_{1}$ and $T_{2}$ are turned off, causing the forward biasing of $D_{3}$ and $D_{4}$ (SIV). S-V and S-VI are similar to S-II and S-III, but relate with the complementary switches and diodes. In this case, turn-on of diodes and turn-off of switches are hard, but the turn-off of diodes and the turn-on of switches are soft. Thus, when the effects of the parasitic components are neglected, inductive load switching may appear to perform better.

Considering the parasitic inductance $\left(L_{p}\right)$ of the converters, figure $3 \mathrm{c}$ shows that the sequence of events is similar to the other conditions discussed earlier. The difference shows up when the transition occurs from S-III to S-IV and the current changes polarity due to the hard turnoff of $T_{1}$ and $T_{2}$, thereby generating a large voltage spike across $L_{p}$ due to current variation. This voltage is added to $v_{L}$ and increases the voltage across switches, which can lead to destruction of the devices.

\subsection{Capacitive load switching $\left(f_{s w}>f_{r}\right)$}

In figure 4, the waveforms and the corresponding sequence of events are shown for the case of switching frequencies greater than the resonant frequency. Observing the figures, one can clearly make out that the current waveform leads the voltage waveform so that this type of commutation is also known as capacitive load switching.

Similar to inductive load switching, the diodes $\left(D_{1}\right.$ and $D_{2}$ ) block the voltage at time interval between the commutation and the change of polarity of $v_{L}$ (at the leading edge of the waveforms before the start of the negative halfcycles). During turn-off interval the load voltage is shared between switches and diodes. This can be observed in figure $4 \mathrm{a}$ and $\mathrm{b}$, where the sum of voltages across $T_{1}$ and $D_{1}$ is equal to $v_{L}\left(=v_{T_{1}}+v_{D_{1}}\right)$. Analysing the sequence of events of figure $4 \mathrm{a}$ and $\mathrm{b}$, in state 'S-I' the current first conducts through $T_{1}$ and $T_{2}$. 


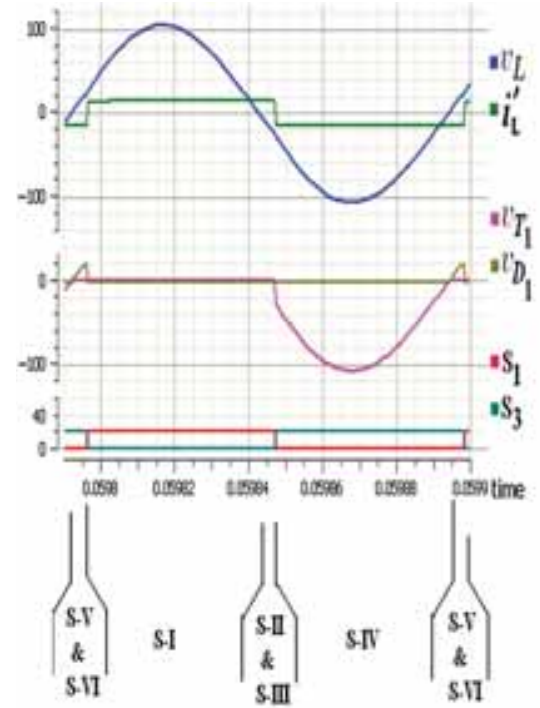

(a)

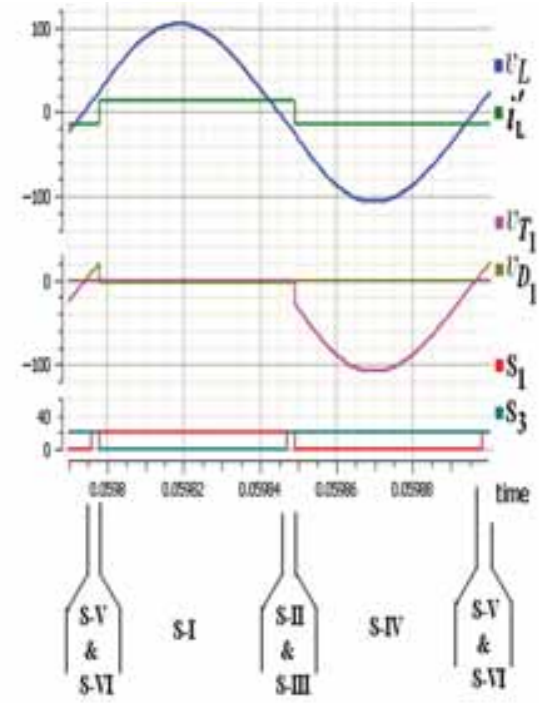

(b)

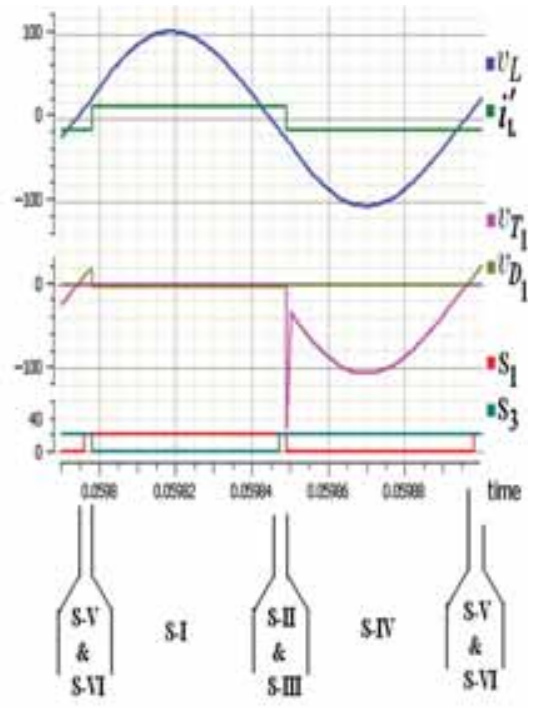

(c)

Figure 3. The waveforms of 'below resonant frequency' operation (a) without considering the parasitic components and overlap time (b) without considering the parasitic components, but considering only overlap time and (c) considering parasitic inductances and overlapping time of the converter.

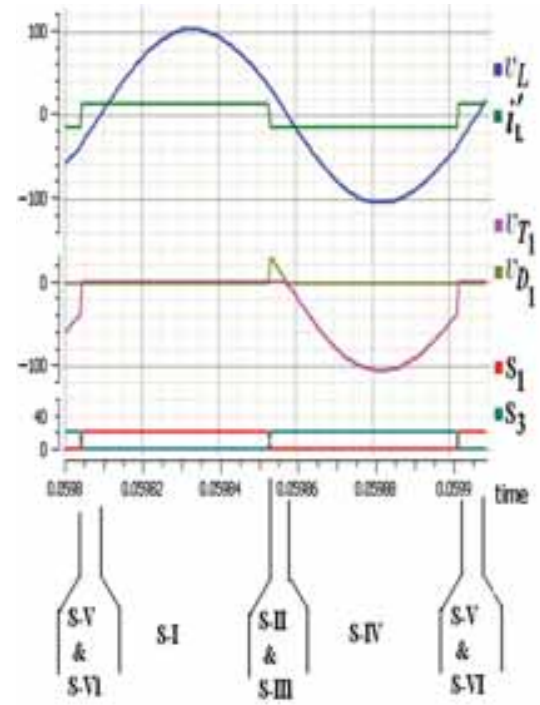

(a)

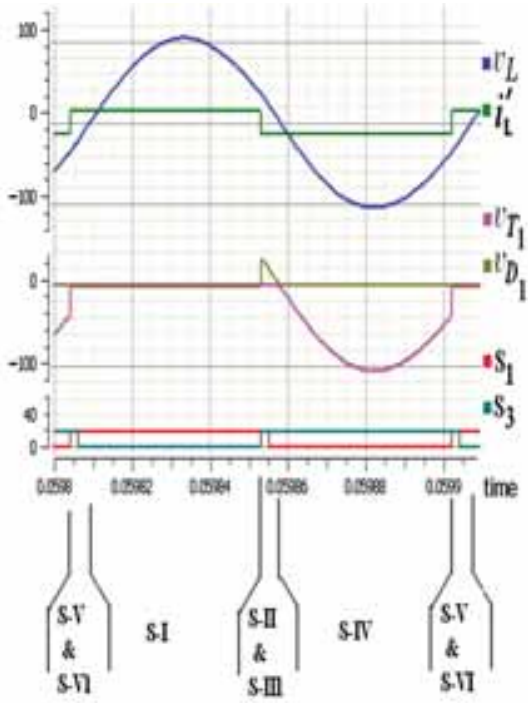

(b)

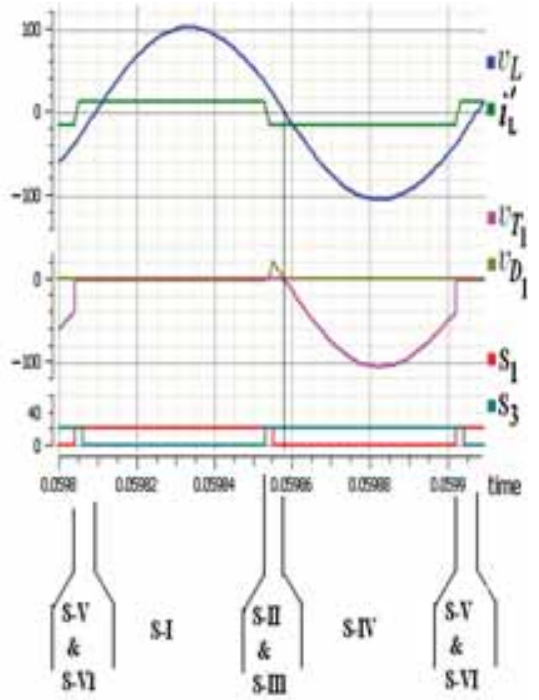

(c)

Figure 4. The waveforms of above resonant frequency operation (a) without considering the parasitic components and overlap time, (b) without considering the parasitic components, but considering only overlap time and (c) considering parasitic inductances and overlapping time of the converter.

Then, $T_{3}$ and $T_{4}$ are turned on while the voltage $v_{L}$ is still positive. Diodes $D_{1}$ and $D_{2}$ are forced to block (off state due to $v_{L}$ polarity), and current conducts through $T_{3}$ and $T_{4}$ (in 'S-II'). Afterwards (during the interval S-III), $T_{1}$ and $T_{2}$ are turned off with ZCS because their series diodes are blocked. Finally, $v_{L}$ changes polarity and diodes $D_{1}$ and $D_{2}$ are forward biased (on state) with ZVS (S-IV). S-V and
S-VI are similar to S-II and S-III, but relate with the complementary switches and diodes. Contrary to the previous case, turn-off of the diodes and turn-on of the switches are hard, but the turn-on of the diodes and the turn-off of the switches are soft. This situation should to be avoided; otherwise series diode recovery currents can create large voltage spikes. 
Considering the parasitic inductance $\left(L_{p}\right)$ of the converters, figure $4 \mathrm{c}$ shows that the voltage $\left(v_{L}\right)$ is shared between switches, diodes and parasitic inductance. The sequence of events is similar to other conditions discussed above. The only difference lies in the parasitic inductance effect on the switching process. Observing the transition between S-I and S-II in figure $4 \mathrm{c}$, considering $L_{p}$, the voltage at the $L_{p}$ terminals opposes the voltage $v_{L}$. Thus, commutation for capacitive switching provides the most acceptable operation of the inverter if one considers $L_{p}$. Although diodes' reverse recovery currents are still a problem, capacitive switching is preferred in CSI.

\subsection{Optimum switching considering parasitic inductances and overlapping time}

In the above sections, different sequences with different operating frequencies for CSI were thoroughly discussed. One may be led to conclude that capacitive switching (switching above $f_{r}$ ) provides more reliable operation for the inverter if $L_{p}$ is considered. We recall that capacitive switching is preferred in CSI. Consequently, to have soft switching during turn-on of diodes and turn-off of switches in capacitive switching, even while considering parasitic inductances and overlapping time, the following sequence of events needs to take place (figures 5 and 6):

1. Switches $T_{1}$ and $T_{2}$ are turned on (and D1 and D2 were already forward biased). During this time interval, load current changes from $-I_{d}$ to $+I_{d}$, and the current in the incoming devices $T_{1}$ and $T_{2}$ rises from zero to $+I_{d}$. Applying $\mathrm{KVL}$ in the loop

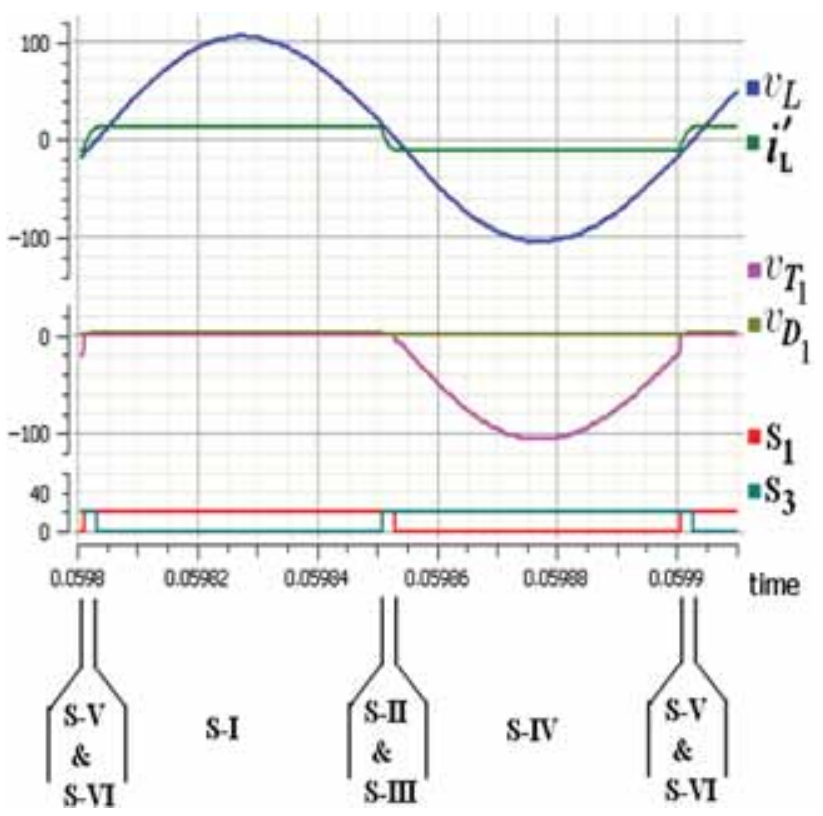

Figure 5. Simulated waveform of induction heating converter at optimum switching frequency.
$D_{1}-T_{1}-A-B-T_{3}-D_{3}-D_{1}$, we realise that the voltage appearing across $T_{1}$ (and $T_{2}$ ) at turn-on is much less in the presence of $L_{p}$. This reduces the turn-on losses and may even take place in a ZVS mode if $v_{T 1}=v_{C}-v_{L p}=0$, which depends on value of the product $L_{p} \frac{d i}{d t}$. In this expression, choice of $d t$ with respect to the measured value of parasitic inductance $L_{p}$ will ensure that $v_{T 1}=0$ at turn-on (ZVS). The relevant derivation is included subsequently.

2. The current $i_{L}^{\prime}$ diminishes and the parasitic inductance $L_{p}$ changes the voltage polarity to maintain the current.

3 . The current $i_{L}^{\prime}$ rises and then equals $I_{d}$ (level), causing the voltage across the inductance $L_{p}$ to be almost zero and diodes $D_{3}$ and $D_{4}$ are blocked by $v_{L}$.

4. Switches $T_{3}$ and $T_{4}$ are turned off with zero current since they are in series with diodes. The load current gets fully transferred to $T_{1}$ and $T_{2}$ as mentioned in point no. 1 above, causing diode $D_{3}$ (and $D_{4}$ ) currents to fall to zero simultaneously (ZCS for both diodes and switches).

5. When half a cycle later, voltage $v_{L}$ changes polarity, $D_{3}$ and $D_{4}$ will get forward biased and will turn on with ZVS. Thereafter the sequence repeats for the complementary switching devices.

Observing figure 5, it may be inferred that conditions for ZVS and ZCS do not occur always. For example, if the overlap duration is low, $T_{3}$ and $T_{4}$ are turned off before $i_{L}^{\prime}$ reaches $I_{d}$ and ZCS does not occur. Another situation arises where the phase shift between current and voltage is low and the voltage changes polarity before $T_{3}$ and $T_{4}$ have turned off. In this case, there is neither ZVS nor ZCS. The combination of $L_{p}, v_{L}$, phase shift and overlap time determines whether soft switching can be achieved or not. This has been established in the subsequent determination.

3.4a Optimum overlap time and phase angle analysis: To find out the optimum switching frequency and the overlap time duration between the gate pulses considering the parasitic inductance in the circuit, the commutation process may be analytically investigated as shown below. Figure $6 \mathrm{a}$ shows the circuit diagram during the commutation from $T_{3} T_{4}$ to $T_{1} T_{2}$, and the corresponding waveforms are shown in figure $6 \mathrm{~b}$. Certain assumptions have to be made to simplify the derivations. Thus, let us assume that the quality factor (here, $Q=14$ ) of the coil is so high that the load voltage waveform is sinusoidal in nature.

Therefore, the load voltage may be written as $v_{L}=\widehat{v}_{L} \sin \omega t$, where, $\widehat{v}_{L}$ is the peak of the load voltage. The converter output current $i_{L}^{\prime}$ leads the load voltage $v_{L}$ by an angle $\phi$. The overlap angle is $\beta$, which corresponds to the time interval during which current $i_{L}^{\prime}$ changes from $-I_{d}$ to $+I_{d}$. In the same time interval a voltage is developed across $L_{p}$ due to this change in current, which is equal to the load voltage $\left(v_{L}\right)$ but in the opposite direction. Hence, in this situation the voltages across the switches are zero if the following condition holds analytically. Taking the instant 


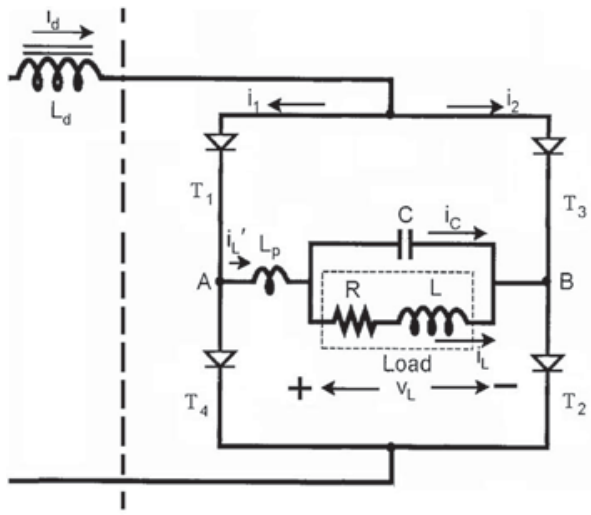

(a)

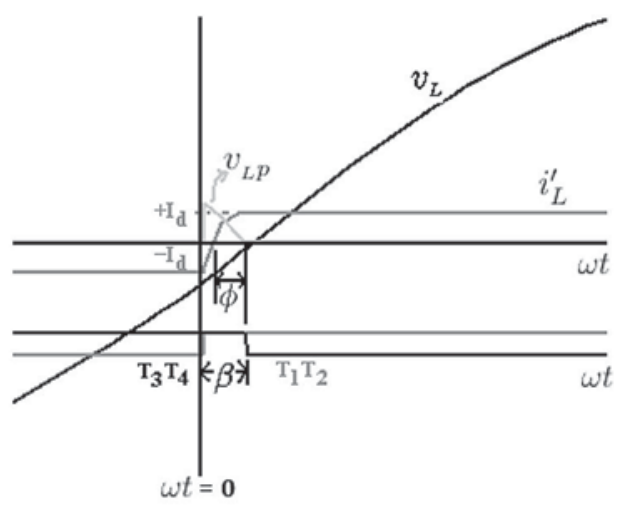

(b)

Figure 6. (a) Circuit diagram of CSI-fed parallel resonant circuit during commutation from $T_{3} T_{4}$ to $T_{1} T_{2}$ and (b) corresponding voltage and current waveforms.

of turn-on of $T_{1}$ and $T_{2}$ as $\omega t=0$, we may write the voltage equation as

$$
\begin{gathered}
v_{L} p(\omega t)=-v_{L}(\omega t)=-L_{p} \frac{d i_{L}^{\prime}(\omega t)}{d t} \\
i_{L}^{\prime}(\omega t)=-\frac{1}{L_{p}} \int v_{L}(\omega t) d t \\
i_{L}^{\prime}(\omega t)=\frac{1}{L_{p}} \frac{\left(-\widehat{v}_{L}\right)}{\omega} \cos \omega t+C
\end{gathered}
$$

where $C$ is the integration constant. At $\omega t=0$, the current $i_{L}^{\prime}(0)=-I_{d}$; therefore, $C=\left(\frac{\widehat{v}_{L}}{\omega L_{p}}-I_{d}\right)$. Now, putting the value of $C$ in Eq. (4), we get

$$
i_{L}^{\prime}(\omega t)=-\frac{\widehat{v}_{L}}{\omega L_{p}} \cos \omega t+\left(\frac{\widehat{v}_{L}}{\omega L_{p}}-I_{d}\right) .
$$

At $\omega t=\beta$, the current $i_{L}^{\prime}(\beta)=+I_{d}$; therefore

$$
\begin{gathered}
i_{L}^{\prime}(\beta)=-\frac{\widehat{v}_{L}}{\omega L_{p}} \cos \beta+\left(\frac{\widehat{v}_{L}}{\omega L_{p}}-I_{d}\right)=I_{d} \\
\beta=\cos ^{-1}\left(1-\frac{2 \omega L_{p} I_{d}}{\widehat{v}_{L}}\right) .
\end{gathered}
$$

Now, again at $\omega t=\beta-\phi$ (figure $6 \mathrm{~b})$, the current $i_{L}^{\prime}(\beta-$ $\phi)=0$ (current zero-crossing leads voltage zero-crossing by $\phi)$. Therefore

$$
\begin{gathered}
i_{L}^{\prime}(\beta-\phi)=-\frac{\widehat{v}_{L}}{\omega L_{p}} \cos (\beta-\phi)+\left(\frac{\widehat{v}_{L}}{\omega L_{p}}-I_{d}\right)=0 \\
(\beta-\phi)=\cos ^{-1}\left(1-\frac{\omega L_{p} I_{d}}{\widehat{v}_{L}}\right)
\end{gathered}
$$

Therefore, the current leads the voltage by

$$
\phi=\beta-\cos ^{-1}\left(1-\frac{\omega L_{p} I_{d}}{\widehat{v}_{L}}\right) .
$$

The optimum overlap and phase difference angle can be found from Eqs. (7) and (10). The combination of $L_{p}, v_{L}$ and $I_{d}$ determines the phase shift and overlap angle to achieve the soft switching in the converter.

Although diode's reverse recovery current is still a problem during capacitive switching, it may be minimised while commutating following the optimum process described above. The current in the diode and the voltage $v_{L}$ are almost zero when the diode is reverse biased. In fact, Jordan et al [10] suggest that commutation be initiated when $i_{L}^{\prime}$ reaches $90 \%$ of $I_{d}$, to avoid the reverse recovery current problem. Under these circumstances, Magraner et al [11] show that the series diodes can be avoided because the parasitic inductance blocks the capacitor's voltage. However, the problems derived from any imprecision in the control strategy can destroy the semiconductors. A control method based on the optimum switching strategy for CSI as presented above finds limited use because of its complexity. Moreover, in case of high value of $L_{p}$ and leading current angle, the reactive power from the source is higher and higher switching frequency is needed, causing an increase in the inverter losses.

\section{Hardware implementation}

The circuit diagram for the induction heating converter has been shown in figure 1 and some photographs of the laboratory prototype are shown in figure 7 . The rectifier module (TSPR40PB), the DC-link capacitor and the buck chopper portion of the converter are clearly seen in figure $7 \mathrm{a}$. Figure $7 \mathrm{~b}$ shows IGBTs with series diodes placed on the heat sink along with the compensating capacitor bank of $21 \mu \mathrm{F}$, while figure $7 \mathrm{c}$ shows the photo of the induction 


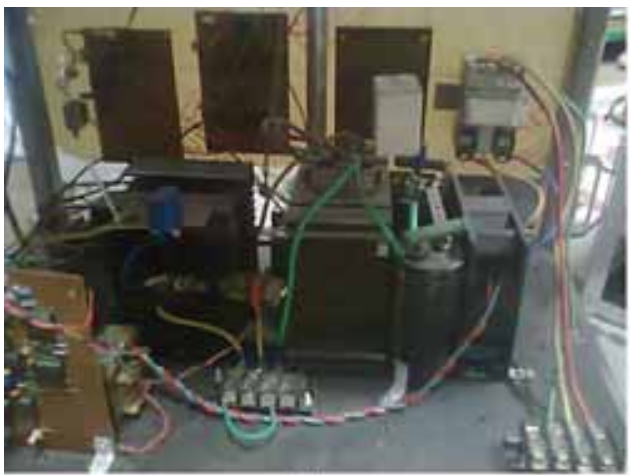

(a)

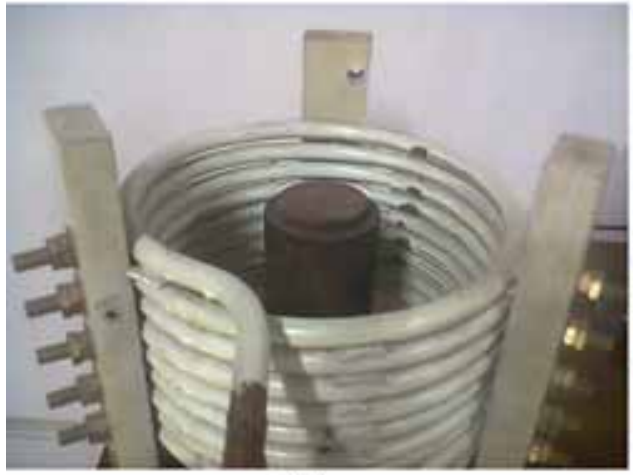

(c)

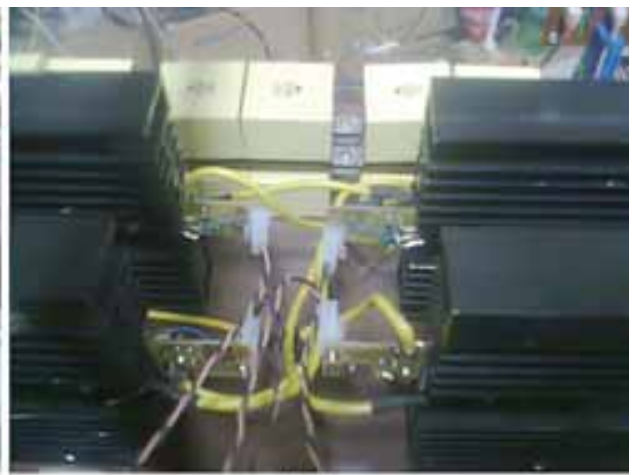

(b)

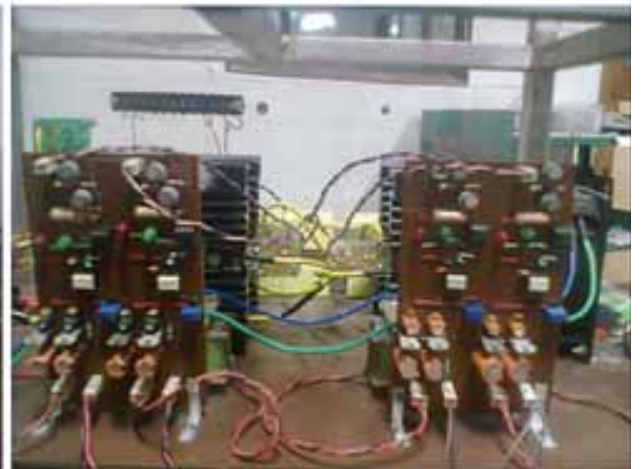

(d)

Figure 7. Photograph of laboratory prototypes of (a) rectifier module and buck chopper, (b) IGBTs with series diode placed on the heat sink and capacitor bank, (c) induction coil and work-piece and (d) IGBT-based converter and its driver circuit.

heating coil and the work-piece (job), which is placed inside the coil. Figure $7 \mathrm{~d}$ shows the IGBT-based converter and its driver circuit. The details of design, fabrication and operation of the entire set-up may be found in [12]. Some pertinent details are given in table 1.

It has been observed that when the load voltage is in phase with the converter current a large voltage appears across the device due to presence of $L_{p}$ (see figure 8a). Figure $8 \mathrm{~b}$ shows the coil and device voltage waveforms when switching frequency is greater than the load-resonant frequency. As discussed in section 3.3 capacitive switching ensures that there is no voltage spike in figure $8 \mathrm{~b}$ even in the presence of $L_{p}$. Switching above $f_{r}$ provides a more

Table 1. Set-up details.

\begin{tabular}{lcc}
\hline Sl. no. & Elements & Value or rating \\
\hline 1 & DC link inductor $\left(L_{d}\right)$ & $20 \mathrm{mH}$ \\
2 & Coil inductance $(L)$ & $15.4 \mu \mathrm{H}$ \\
3 & Coil resistance $(R)$ & $54 \mathrm{~m} \Omega$ \\
4 & Capacitor across coil $(C)$ & $21 \mu \mathrm{F}$ \\
5 & IGBT & IRG4PC40U \\
6 & Diode & DSEI60-12A \\
7 & Overlap time & $1.2 \mu \mathrm{s}$ \\
8 & Parasitic inductance $\left(L_{p}\right)$ & $0.03 \mu \mathrm{H}$ \\
\hline
\end{tabular}

reliable operation of the CSI. However the spike is clearly visible in case of inductive switching, shown in figure $8 \mathrm{c}$.

The experimental waveforms of the load voltage and inverter terminal current have been shown in figure 9a when the converter is operated at optimum switching frequency. It is seen that the current waveform is just a little leading $\left(2.8^{\circ}\right.$ whereas the calculated value of angle $\phi$ is $2.7^{\circ}$ ), which is thus due to a frequency just above resonant frequency when current would have been in phase with voltage. It is clearly seen from the device voltage waveform in figure $9 \mathrm{~b}$ that soft switching takes place during turn-on of diodes and turn-off of switches. The calculated resonant frequency is around $8.86 \mathrm{kHz}$ for the present billet sample in this set-up.

\section{Conclusions}

In this paper, existing commutation strategies have been reviewed for application in the current-source-inverter-fed resonant circuit with unidirectional current switches with bidirectional blocking voltage capability, implemented with switches (IGBT with series diode), keeping IH applications in mind. The factors that need to be considered in the selection of the appropriate strategy are the working frequency and the switching characteristics of the IGBTs used. 


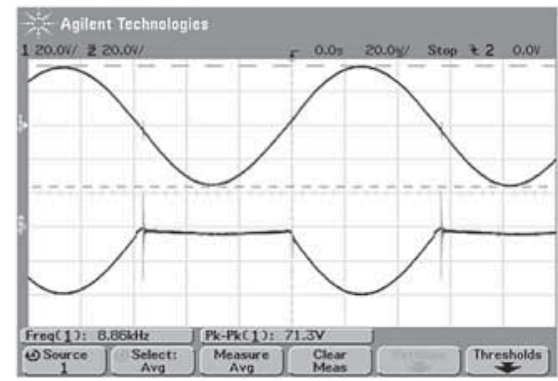

(a)

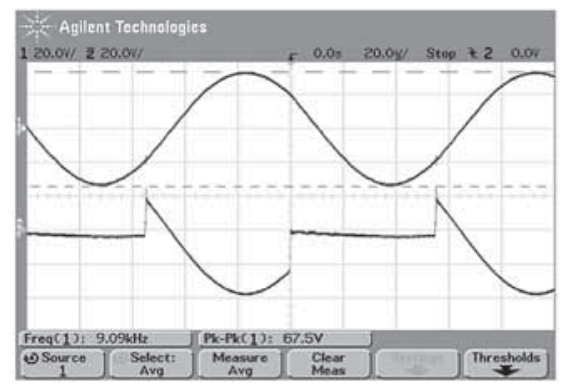

(b)

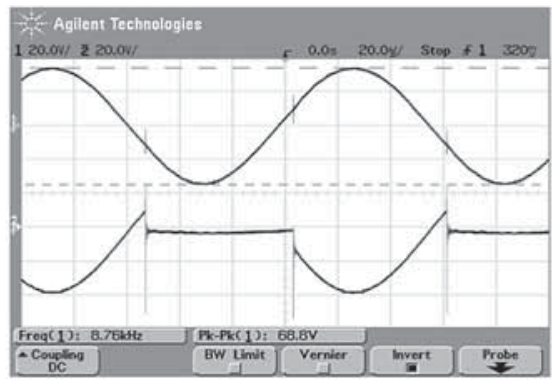

(c)

Figure 8. Experimental waveform of laboratory-made induction heating converter at three different switching frequencies. (a) CH1: coil voltage (20 V/div); CH2: device voltage (20 V/div) at resonant frequency switching. (b) $\mathrm{CH} 1$ : coil voltage (20 V/div); CH2: device voltage $(20 \mathrm{~V} / \mathrm{div})$ at capacitive switching. (c) $\mathrm{CH} 1$ : coil voltage $(20 \mathrm{~V} / \mathrm{div})$; $\mathrm{CH} 2$ : device voltage $(20 \mathrm{~V} / \mathrm{div})$ at inductive switching.

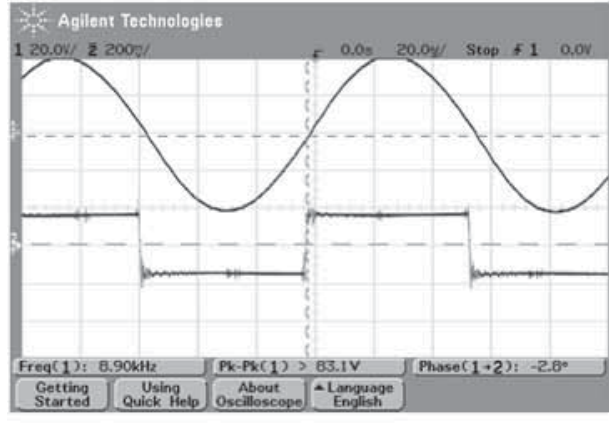

(a)

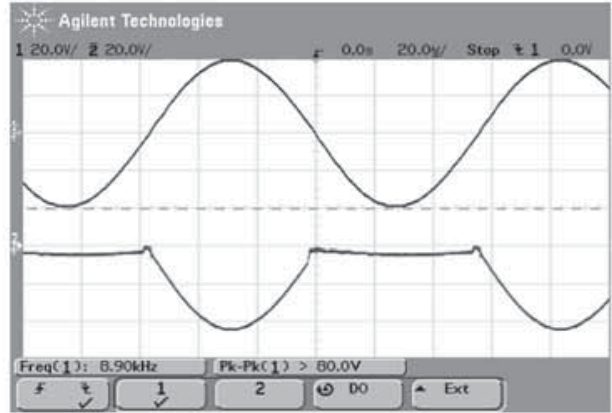

(b)

Figure 9. Experimental waveform of laboratory-made induction heating converter at optimum switching frequency. (a) CH1: coil voltage (20 V/div); $\mathrm{CH} 2$ : inverter terminal current (4A/div). (b) $\mathrm{CH} 1$ : coil voltage (20 V/div); $\mathrm{CH} 2$ : device voltage (20 V/div).

A switching frequency just above the load-resonant frequency and a precisely calculated overlap time that can neutralise the effect of the parasitic inductance is the perfect combination for smooth and fault-free operation of a CSI used in IH applications. Accurate experimental results of tests conducted with a laboratory-fabricated $2 \mathrm{~kW}$ prototype provide sound verification of the above claims. The practical results are found to be in excellent correlation with simulated waveforms.

\section{Acknowledgements}

The authors wish to thank M/S EMT Megatherm Pvt. Ltd., Kolkata, for the technical support in making the induction heating coils. The support and encouragement received from Late Prof. Ajit K Chattopadhyay, Emeritus Professor, Department of Electrical Engineering, IIEST, Shibpur, is gratefully acknowledged. The authors also acknowledge the support received from the authorities of IIEST, Shibpur, Howrah, and particularly from research colleagues at the Advanced Power Electronics Laboratory, Department of Electrical Engineering, towards this work.

\section{References}

[1] Roy S 2007 Finite element based design, fabrication and tasting of a $2 \mathrm{~kW}, 20 \mathrm{~A}, 10 \mathrm{kHz}$ CSI fed single phase induction furnace for application in bar/billet heating. $\mathrm{ME}$ thesis, BESU, Shibpur, India

[2] Chudnovsky V, Axelrod B and Shenkman A 1996 Medium frequency combined inverter for electroheat with improved starting arrangements. IEE Proc. Electr. Power Appl. 143(2): 165-171

[3] Dawson F P and Jain P 1991 A comparison of load commutated inverter systems for induction heating and melting applications. IEEE Trans. Power Electron. 6(3): 430-441

[4] Dede E J, Gonzilez J V, Linares J A, Jordan J, Ramirez D and Rueda P 1991 25-kW/50-kHz generator for induction heating. IEEE Trans. Ind. Electron. 38(3): 203-209

[5] Frank W E 1970 New developments in high-frequency power sources. IEEE Trans. Ind. Gen. Appl. IGA-6(I): 29-35

[6] Pelly B R 1970 Latest developments in static high frequency power sources for induction heating. IEEE Trans. Ind. Electron. Contr. Instrum. IECI-17(4): 297-312

[7] Mohan N, Undeland T and Robbins W 2006 Power electronics: converters, applications, and design. London: Wiley

[8] Roy M and Sengupta M 2016 Design, real time modeling, simulation and digital implementation of phase-locked loop 
based auto-synchronizing current sourced converter for an induction heating prototype. Sadhana (accepted)

[9] Kerchner R M and Corcoran G F 1960 Alternating-current circuits. New York: Wiley

[10] Jordan J, Esteve V, Dede E, Cases C, Magraner J M, Sanchis E, Maset E, Ferreres A and Ejea J 2007 Automated calculation method to determine the output power based on the frequency for induction heating IGBT parallel inverter. Power Electronics Specialists Conference Proceedings, pp. 713-718
[11] Magraner J M, Jordan J, Cases C, Esteve V, Dede E, Sanchis E, Maset E, Ferreres A, Ejea J B and Gumbau E 2009 Firing strategy for bidirectional current switches with unidirectional voltage blocking capability in induction heating parallel resonant inverters. Proceedings of the 13th European Conference on and Power Electronics and Applications, pp. 1-7

[12] Roy M and Sengupta M 2012 Design, fabrication and testing of two different laboratory prototypes of CSI-based induction heating units. J. Inst. Eng (India) Ser. B 93(3): 133-141 